\title{
Agomelatine Addressing Behavioural Facets in Autism
}

\section{Ahmed Naguy ${ }^{1 *}$ and Ali Al-Tajali ${ }^{2}$}

${ }^{1}$ Child \& Adolescent Psychiatrist, Kuwait Centre for Mental Health (KCMH), Kuwait ${ }^{2}$ General Adult Psychiatrist, Head of Neuromodulation Unit, KCMH, Kuwait

Keywords: Challenging behaviours; Autism; Agomelatine

\section{To the Editor}

Autism Spectrum Disorder (ASD) is usually coupled with a multitude of problem behaviours including ADHD-like symptoms (31-50\%), tics (22\%), mood/anxiety symptoms (30-40\%) and aberrant eating/sleeping patterns [1]. These challenging behaviours are primarily a cause of referral to child psychiatrists. When psychosocial and educational interventions are inadequate, pharmacotherapy is indicated [2]. Both atypical antipsychotics, risperidone (Risperdal ${ }^{\circ}$ ) and aripiprazole $\left(\right.$ Abilify ${ }^{\circ}$ ) are currently FDA-approved to address these behavioural facets [3]. Nonetheless, these agents, along with other antipsychotics, are plagued with a myriad of adverse drug reactions, notably, the neurologic and cardio-metabolic syndromes [4]. This amply illustrates a pressing need to explore alternative agents that are both effective and tolerable. Here we are reporting a case of ASD where the atypical antidepressant agomelatine (Valdoxan ${ }^{\circledR}$ ) favourably tackled associated behavioural facets with great tolerability.

A 10-year-old male Kuwaiti kid was casualty petitioned by his parents for behavioural dyscontrol. He was long diagnosed as ASD, according to DSM-5, with mild intellectual disability, non-verbal but with no seizure activity attending special schooling. As reported, he is hyperactive, yelling and screaming, cranky, with continuous body rocking movements and disturbed sleeping in the form of difficulty falling asleep and frequent nocturnal awakenings. This is now escalating in a crescendo manner. He was meticulously scrutinized for medical and environmental causations that could be contributory to this behavioural decompensation with negative yield. Past psychotropic meds history includes intolerance to both risperidone (incurred acute dystonic reactions, hyperprolactinaemia and weight gain) and aripiprazole (incurred severe akathisia) with mediocre response at best, as reported by parents. Baseline LFTs were normal. We opt for agomelatine to target sleep problems which parents found most troublesome. Agomelatine $25 \mathrm{mg}$ at bedtime was prescribed. At week 3, unexpectedly, there was a tangible improvement both clinically and objectified by marked reductions of hyperactivity (39 before treatment/17 after treatment), irritability (36 before/16 after) and stereotypies (16 before/7 after) subscales of Aberrant Behaviour Checklist (ABC), with concomitant normalization of sleep pattern. Response was well-maintained at W-6 and W-12 with follow-ups. No major adverse drug reactions were reported during the trial. Agomelatine is atypical antidepressant with a novel mechanism of action, namely, 5HT2C antagonism and M1 \& M2 melatonergic agonism or colloquially MASSA (melatonergic agonist and selective serotonergic antagonist) [5]. This would translate, psychopharmacologically, into noradrenaline-dopamine disinhibition (NDDI) and a chronobiotic actions, respectively. NDDI might help with hyperactivity, akin to stimulant actions in ADHD. 5-HT2C antagonism is ultimately proserontonergic at 5HT1A and thus antidepressant, anxiolytic and antiobsessional. Melatonergic actions tend to synchronize circadian rhythms without compromising daytime vigilance. Agomelatine has a benign side effects profile but mandates periodic liver function monitoring [6]. This case report shows agomelatine could be an appealing addition to the psychopharmacologic armamentarium addressing behavioural facets in ASD. This goes in tandem with a case report of autistic dermatillomania that responded favourably to agomelatine [7]. Contrariwise, Niederhofer found no superiority of agomelatine over other antidepressants in ASD [8].

\section{References}

1. Leyfer OT, Folstein SE, Bacalman S (2006) Comorbid Psychiatric Disorders in Children with Autism: Interview Development and Rates of Disorders. J Autism Dev Disord 36: 849-861.

2. Palermo MT, Curatolo $P$ (2004) Pharmacologic treatment of autism. J Child Neuro 19: 155-164.

3. Posey DJ, Stigler KA, Erickson CA (2010) Antipsychotics in the treatment of autism. J Clin Invest 118: 6-14.

4. Pramyothin P, Khaodhiar L (2010) Metabolic syndrome with the atypical antipsychotics. Curr Opin Endocrinol Diabetes. Curr Opin Endocrinol Diabetes Obes 17: 460-466

5. Stahl SM (2014) Mechanism of action of agomelatine: a novel antidepressant exploiting synergy between monaminergic and melatonergic properties. CNS Spectr 19: 207-212.

6. Maclsaac SE, Carvalho AF, Cha DS (2014) The mechanism, efficacy, and tolerability profile of agomelatine. Expert Opin Pharmacoth 15: 259-274.

7. Antoniadis D, Floros GD, Nikolaidis N (2013) Response to agomelatine: treatment of an obsessive skin picking episode. Ann Clin Psychiatry 25: 228-229.

8. Niederhofer $\mathrm{H}$ (2011) Efficacy of duloxetine and agomelatine does not exceed that of other antidepressants in patients with autistic disorder: preliminary results in 3 patients. Prim Care Companion CNS Disord 13.
*Corresponding author: Ahmed Naguy, Kuwait Centre for Mental Health, Shuwaikh, State of Kuwait, Tel: 96565541937; E-mail: ahmednaugy@hotmail.co.uk

Received March 12, 2015; Accepted April 21, 2015; Published April 28, 2015

Citation: Naguy A, Al-Tajali A (2015) Agomelatine Addressing Behavioural Facets in Autism. J Psychiatry 18: 283 doi: 10.4172/2378-5756.1000283

Copyright: ( 2015 Naguy A, et al. This is an open-access article distributed unde the terms of the Creative Commons Attribution License, which permits unrestricted use, distribution, and reproduction in any medium, provided the original author and source are credited 